\title{
Espaço, Tempo e Estruturas das Teorias do Movimento
}

\author{
Space, time and structures of motion theories
}

\author{
Ademir Eugenio de Santana*10 \\ ${ }^{1}$ Universidade de Brasília, Brasília, DF, Brasil
}

Recebido em 17 de Maio, 2018. Revisado em 23 de Julho, 2018. Aceito em 26 de Julho, 2018.

\begin{abstract}
Considerando a conceitualização e definição do tempo e do espaço, elementos constitutivos de um programa mecânico, ou seja, de uma teoria do movimento, são identificados. Primeiro, aspectos históricos sobre a mensuração e a geometrização do conceito de tempo e espaço são descritos. A análise sobre os projetos mecânicos é então desenvolvida a partir da definição de pontos inerciais (materiais) e de campos. Como aplicação desse método, a mecânica clássica nas suas versões relativísticas e não-relativísticas são discutidas, com ênfase na natureza do tempo. Como outro exemplo, a teoria cinética é estudada enquanto uma teoria de campo. Aspectos pedagógicos sobre a transposição didática dos conceitos abordados são apresentados, em particular no caso da teoria cinética. Palavras-chave: Tempo, Espaço, Teorias do Movimento
\end{abstract}

Considering a conceptualization and a definition of time and space, constitutive elements of a mechanical program, that is, of a motion theory, are identified. Historic elements regarding measurement and geometric aspects of time and space are described. The analysis of mechanical theories is carried out by considering a definition for inertial points and fields. As an application of this approach, the relativistic and the non-relativistic mechanics are analysed, emphasizing the nature of time. As another example, the relativistic kinetic theory is studied as a field theory. Pedagogical aspects regarding a didactic transposition of concepts are presented, in particular with the formalism for the kinetic theory.

Keywords: Time, space, Theories of Motion

\section{Introdução}

A análise comparativa de teorias do movimento, ou seja a axiomatização, conceitualização e definição de limites de aplicabilidade das mecânicas, é uma tarefa de longa data que se notabiliza com a postulação da relatividade restrita e da mecânica quântica. A importância deste tipo de estudo, mesmo que inicialmente motivado por questões acadêmicas, e não por problemas de aplicações imediatas [1 7], conduziu a significativas descobertas de natureza conceitual e prática. Um exemplo notório foi a proposição das desigualdades de Bell [8, 9], associadas ao desenvolvimento subsequente de conceitos como emaranhamento e teletransporte de estados da radiação; noções fundadoras para a computação e comunicação quânticas 10 15.

Esse tipo de análise permance relevante, quer seja no contexto inicial, com a comparação entre a teoria quântica e a mecânica clássica (ambas não relativísticas), como também ganha novos domínios devido à consagração do modelo padrão da física de partículas elementares, com a detecção do bósons de Higgs 16], e da relatividade geral, com a mensuração do gráviton [17]. Ou seja, inclui a teoria quântica de campos que, ao apontar para possibilidades amplas de novos fenômenos físicos, exige uma

*Endereço de correspondência: asantana@unb.br conceitualização, tanto matemática quanto física, a partir de bases sólidas: um problema ainda em aberto [18 20].

A questão se torna mais intricada na perspectiva pedagógica, pois demanda um processo de transposição didática, no qual os elementos a serem transpostos encontram-se em desenvolvimento 18 20]. Por exemplo, um campo quântico, intrinsecamente ligado à noção de partícula quântica elementar, se, na perspectiva matemática, está satisfatoriamente apresentado nos livros canônicos dessa disciplina 21], conceitualmente, permanece por vezes como um objeto de entendimento físico difuso, pois, em muitos casos, misturam-se noções aparentemente antagônicas, como a de ponto inercial (material) e de amplitude de probabilidade.

A função de onda em mecânica quântica é interpretada corretamente em bons livros 22. como uma amplitude de probabilidade, caracterizada como um objeto estendido no espaço descrevendo um sistema físico como um elétron. Ou seja, a função de onda é um campo clássico. Em outras situações, contudo, mesmo em tentativas axiomáticas, essa amplitude é embrulhada em um pacote junto com a noção de ponto material [23]; e disso emergem limitações conceituais e muita dificuldade pedagógica. Há de se considerar também que muitos outros objetos mecânicos foram introduzidos nas últimas décadas e demandam amadurecimento conceitual, para que sejam explorados na plenitude. Assim são as noções de cor e de confinamento/deconfinamento da matéria hadrônica $21,24,25]$. 
Um elemento importante nessa análise é a caracterização de uma teoria do movimento; ou de modo outro, de uma teoria mecânica. Axiomaticamente, esse problema tem sido considerado, por exemplo, pelas teorias de representação de grupos de simetrias. Este programa foi iniciado por Wigner 26, 27, e que, junto aos teoremas de Noether, encontra apogeu nas teorias de calibre abeliano e não-abeliano; ou seja, na edificação do modelo padrão da física de partículas 19 21. Mas também neste caso, nem todos os elementos estruturantes ficam completamente estabelecidos de imediato, e o problema da conceitualização vem sendo desenvolvido e explorado tanto a partir de bases teórico-axiomáticas quanto experimentais, mas que distante está de ser completamente resolvido 28,29 . Tomando por substrato resultados prévios 30 35, o propósito do presente trabalho é abordar a questão de construção de teorias mecânicas, mas a partir de uma perspectiva histórico-pedagógica e das bases empíricas da física. De início, as definições de tempo e de espaço 31 são introduzidas como asseguradas pela práxis experimental da física 36 ; e assim segue uma discussão sobre a natureza de um sistema físico, sobre o conceito de estado mecânico e sobre as relações de causalidade que levam às equações de movimento 34 . Após a identificação dos elementos constitutivos de uma teoria mecânica [31,34,35], como exemplo, a mecânica clássica relativística e não relativística são analisadas tanto quanto possível pedagogicamente. Entretanto, a conceitualização apresentada se presta para a análise de outras teorias do movimento, como a mecânica quântica e a teoria quântica de campos 35. Neste sentido, como aplicação, a teoria cinética relativística é formulada como uma teoria de campos, a partir de uma Lagrangiana invariante por transformações de Lorentz. Este não é um procedimento usual para se iniciar a teoria cinética, mas se revela importante para propósitos pedagógicos e para a discussão conduzida aqui.

O trabalho está organizado da seguinte forma. Na seção 2, elementos históricos sobre a mensuração e a geometrização do conceito de tempo e espaço são descritos. Na seção 3, as bases teóricas e empíricas sobre o tempo são revisitadas, de modo que, na seção 4, tendo em vista a construção de teorias do movimento, as definições de tempo, espaço e sistemas inerciais são apresentadas. Na seção 5, os sistemas mecânicos são caracterizados por meio de conceitos primitivos de ponto material e de campo. Na seção 6, os elementos constitutivos das teorias mecânicas são estabelecidos, e a mecânica clássica nas suas versões relativísticas e não relativísticas são discutidas, com ênfase na conceitualização do tempo. $\mathrm{Na}$ seção 7, como aplicação, a teoria cinética relativística é abordada enquanto uma teoria de campos clássicos. As conclusões finais estão apresentadas na seção 8.

\section{Mensuração e geometrização do Tempo: aspectos históricos}

Para se abordar a questão do tempo nas teorias físicas, dois aspectos são centrais: os processos empíricos, en- quanto elementos definidores da noção de tempo, e os geométricos, associados a atributos qualitativos teóricos; e este tem sido o caminho histórico.

A noção de tempo, seu desenvolvimento sistemático e pragmático, através do instrumento de medida, o relógio, está, para uns, na essência do processo que levou à hegemonia cultural, científica e econômica da sociedade ocidental 37. Esse desenvolvimento é reminescente dos primórdios da idade média e encontra a primeira síntese na mecânica proposta por Newton 38. É a partir de então, com a matematização do conceito de tempo associada ao desenvolvimento das mecânicas e das técnicas 34, que se chegou ao relógio atômico, que utiliza as vibrações de cristais de quartzo com frequência em torno de 2,5 megaciclos por segundo, ajustado por um sistema ressonante de Césio vibrando a 9.192.631.770 \pm 20 ciclos por segundo.

O controle e a apropriação do tempo, no decorrer do processo histórico denominado civilizatório, estiveram sempre atrelados ao poder e a riqueza. Na China antiga, por exemplo, um calendário era um pré-requisito à soberania 39 41, e cada imperador costumava marcar seu período com a promulgação de um novo calendário. Desse modo, era possível reger as atividades diárias (principalmente nas vilas), desde as dos soldados, às dos artesões; prever os períodos de colheita ou plantio; promulgar datas dos rituais religiosos; um solstício deveria ser antecipado com certa acurácia. Esse tipo de controle se constituía em especial segredo de estado, e o imperador, por sua vez, garantia a legitimidade de suas decisões, harmonizadas com os padrões cósmicos. Todavia, seu poder (Crônico) emanava dos conhecimentos acumulados pelos astrônomos (ou astrólogos), sempre um seleto e influente grupo na corte.

Na busca de acurácia, os chineses desenvolveram os relógios de água (clepsidras), chegando a uma maravilha no século XI (durante a dinastia Sung): o famoso relógio de $\mathrm{Su}$ Sung (uma réplica de um tal relógio se encontra no museu do tempo em Rockford), desenvolvido para reproduzir o movimento de três luninescências: o Sol a Lua e (um conjunto de) estrelas, elementos básicos da divinação astrológica chinesa, registrando o efêmero erro, para a época, de um minuto ao dia. Esse grau de precisão foi alcançado na Europa somente no século XVII, quando Huygens construiu seu relógio de pêndulo, primeiro proposto por Galilei [40].

Conceitualmente, dividir o tempo em unidades e medilas através de comparações de fenômenos entre si - isto é, via relações ou processos, foi uma conquista significativa. Mas não menos radical e revolucionária, foi a invenção do relógio mecânico, que surgiu durante a idade medieval européia. Embora se desconheça o inventor, Whitrow 41] especula que o relógio mecânico pode ter sido desenvolvido dentro dos monastérios europeus, como uma maneira de manter o estímulo à rigidez da disciplina monástica (nos monastérios chineses isso era resolvido com a queima de varetas de incenso).

O relógio mecânico é um aparato concebido a partir de molas e engrenagens e, principalmente, de movimentos 
repetitivos; diferentemente dos relógios do tipo clepsidras baseados no fluxo contínuos de água. O potencial tecnológico de um tal aparato mecânico residia na possibilidade de miniaturização e, como consequência, a apropriação de uso pessoal. A partir de então, o relógio, que nunca amordaçou Cronos, estabeleceu, como lastimam alguns, à lâmina da espada, sua tirania sobre o ocidente. De fato, o relógio mecânico foi essencial para o processo que levou a revolução industrial e idade contemporânea 41].

Por exemplo, no contexto histórico-econômico, a descoberta do cronômetro marítmo foi definidora para a hegemonia britânica sobre o mercantilismo avançado e a revolução industrial [37]. Com esse dispositivo foi possível encontrar a solução para a questão da localização na longitude, problema este que freiou o domínio completo dos mares por, pelo menos, três séculos.

O problema da longitude 42,43 consistiu na busca de um meio seguro para se estabelecer com precisão a localização na longitude terrestre (o problema da latitude já havia sido resolvido séculos antes com a bússola [37,42]). A lista de nomes envolvidos, um notável relicário de celebridades científicas, encerra a importância e a dificuldade do problema: Galilei, Kepler, Newton, Huygens, Euler, Laplace, Lagrange, dentre outros em toda a Europa. Vários prêmios foram oferecidos, por várias cortes, para quem apresentasse uma solução viável. Vale lembrar a criação do Conselho da Longitude em 1714 na Inglaterra, oferecendo um prêmio de $\mathfrak{L} 20.000$ (atualmente, da ordem de US $\$ 12$ milhões). O prêmio foi dado em 1773 a John Harrison, um relojoeiro com formação em carpintaria. $\mathrm{O}$ fato de ser um bom conhecedor de madeira foi o que permitiu a Harrison suplantar, num trabalho de décadas, as dificuldades com atrito, dilatação e desgaste de metais; dificuldades típicas em uma engrenagem da época, principalmente quando submetidas às intempéries dos oceanos.

A longa história de Harrison e a solução encontrada estão descritas em ampla literatura [37,42]. Entretanto, um elemento básico de sua solução: Harrison assumiu como pressuposto (conhecido, mas inviável até então) que o tempo serviria como definidor de dimensões espacias uma perpectiva espaço-geométrica do tempo: a cada 4 minutos de rotação da Terra correspondem a $1^{\circ}$ de arco de longitude em todo o globo, que se traduz em 109,4 km no Equador. Bastava, então, relógios sincronizados, um em terra e outro na embarcação. Conhecendo o tempo local, onde a embarcação estava, bastaria consultar o relógio de bordo, sincronizado ao de terra, para saber a diferença de tempo, e portanto quantos graus de longitude. Esta possibilidade geométrica para o tempo foi proposta em 1530, pelo astrônomo flamengo Gemma Frisius, que defendia o uso do relógio mecânico para tanto. A idéia de usar relógios sincronizados solucionaria o problema de modo teórico simples, mas esbarrava na falta de técnica para a construção de relógios de precisão. O próprio Newton, um dos fundadores do Conselho da Longitude, descartava essa possibilidade como viável [37,42]. E se o status quo acadêmico do século XVIII remetia a uma solução associada aos astros, Harrison assumiu a postura contrária, e passou a considerar a perspectiva espaço-temporal geométrica de Frisius.

Essa perspectiva geométrica do tempo que se desenvolveu na Europa, de um ponto de vista teórico-filosófico, teve sua origem na Grécia. Foi Aristóteles quem primeiro estabeleceu que tempo era movimento. Não obstante as várias críticas, essa proposta tem sido efetivamente adotada pela ciência desde Newton, que estabeleceu a primeira matematização rigorosa sobre o tempo [40].

Que, com essa matematização, Newton fez pelo tempo o que os gregos fizeram com o espaço - um tipo de geometrização - é um fato estabelecido; como também é o reconhecimento de que novos elementos associados às propriedades do tempo apareceram com a física moderna, em particular, com Einstein. Entretanto, persistem controvérsias no entendimento e no papel que cada uma dessas teorias desempenham na compreensão do tempo. Outrossim, ainda se requer o estabelecimento claro e preciso das diferenças, semelhanças, inovações e abrangência de significado do tempo em sua apropriação pela física $[44-46]$. Esse é o caso da dificuldade encontrada pela física ao abordar o problema da irreversibilidade [47 49]. Sob um olhar mecânico estrito, podemos então procurar entender e comparar o impacto da física moderna na conceituação de tempo.

\section{Bases teórico-empíricas do tempo}

Se o ato de compreender é também catalogar, classificar, distinguir ao criar nomenclatura própria e prover respostas a questões estabelecidas, mesmo que de modo preliminar, então foi Santo Agostinho quem abriu a trilha da sistematização para o estudo do tempo, indo na direção de uma mecânica matematicamente edificada. Em suas Confissões, Livro XI [50], Agostinho trata primeiro de responder a questões teológicas do tipo: "Que fazia Deus antes de Criar o céu e a terra? "; responde, então, com certo estilo lacônico e pragmático,"Mas eu digo meu Deus, que sois o criador de tudo o que foi criado,.... Os vossos anos não vão nem vêm. Porém, os nossos vão e vêm, para que todos venham. Todos os vossos anos estão conjuntamente parados, porque estão fixos, nem os anos que chegam expulsam os que vão, porque estes não passam. Quanto aos nossos anos, só poderão existir todos, quando já todos não existirem... ". E então, com o tempo no reino das criaturas, e não no dos demiurgos, o Santo esvazia a questão inicial: "Não houve tempo nenhum em que não fizésseis alguma coisa, pois fazíeis o tempo".

A partir de então, Agostinho perfaz sua sistemática, discutindo a noção de passado, futuro, de tempo longo, breve; o conceito de tempo que flui do futuro para o presente desaguando no passado; e a quesão da mensuração. Para Agostinho, "...medimos os tempos que passam, de modo que podemos afirmar: este espaço de tempo é duplo de tal outro, ou é-lhe equivalente, ou este é o igual àquele... . Por conseguinte, medimos os tempos ao decorrerem. E se alguém me disser: 'Como sabeis?', responder-lhe-ei: 'Sei-o porque o medimos'. Não medimos o que não existe. Ora, as coisas pretéritas ou futuras 
não existem. Como medimos nós o tempo presente, se não tem espaço [intervalo]". Mais uma vez a resposta denuncia o estilo: "Mede-se quando passa".

Agostinho funda, então, uma tradição com Boethius, Anselmo, entre outros, com Deus exististindo fora do tempo. A despeito de detratores e defensores, dentro e fora da Igreja, um aspecto importante na perspectiva de Agostinho é a quase matematização de seu conceito de tempo via o processo de mensuração. Desse modo, a caracterização do tempo ocorre pela medida, estabelecida por sua vez pela comparação de processos 40. É esta mensuração que será utilizada, quase uma dúzia de séculos mais tarde, primeiro por Galilei de um ponto de vista prático, e depois por Newton, na Europa renascentista, encerrando uma axiomatização da mecânica.

Newton lança os fundamentos da mecânica moderna no célebre Philosophiae naturalis principia mathematica [38]. Inicia o Livro I com uma seção de definicões cuidadosas de conceitos, com certo estilo Agostiniano revelador:

Definição I - Principia (Livro I): The quantity of matter is the measure of the same, arising from its density and bulk conjunctly

Definição II - Principia (Livro I): The quantity of motion is the mesure of the same, arising from the velocity and quantity of matter conjunctly.

Sobre o tempo, estabelece 38]:

"Absolute, true, and mathematical time, of itself, and from its own nature flows equably without regard to anything external, and by another name is called duration; relative, apparent, and common time, is some sensible and external (whether accurate or unequable) measure of duration by means of motion, which is commonly used instead of true time; such as an hour, a day, a month, a year."

Não obstante Newton assumir uma concepção filosófica de um tempo absoluto, um ente de existência objetiva própria, independente dos processos, é a abordagem realista dos processos temporais, via fenômenos físicos, que é usada e matematizada, de fato, nos Principia. A formulação de Newton deve então ser depurada a partir de seus elementos básicos. Ou seja, uma síntese de programa mecânico, deve ser estabelecida a partir das características do conceito de tempo nas diversas mecânicas existentes, incluindo a de Newton e a relativística. Nesse sentido é necessário primeiro decodificar o procedimento de mensuração em física, que se estabelece com os sistemas de referência.

\section{Definição de espaço, tempo e sistemas de Referência inerciais}

Os fenômenos físicos (ou mecânicos) são caracterizados a partir de sistemas de referência inerciais (SIs), que constituem um conceito primitivo, sendo definidos pelo fato de que neles se estabelecem os laboratórios de observação, de modo que as leis da mecânica sejam invariantes. A partir de tais sistemas, especifica-se, para os eventos, o espaço, definido por réguas, e o tempo, definido por relógios. Os elementos ontológicos na caracterização desses conceitos estabelecem uma visão realista da física, através da qual os objetos do mundo estão dados, existem como são, $a$ priori, e entre eles há relações (ou processos) e movimento, como noções também primitivas. Ressalte-se que não há tempo, nem espaço, e portanto não há física, para um universo onde só exista um único objeto, sistema, físico. Nesse caso, nem mesmo a noção de movimento faria sentido.

Utilizando-se o conceito de relações (ou processos), estabelecem-se padrões. Assim é introduzida por exemplo a noção de distância. Com a escolha de um padrão - a régua -, compara-se um objeto com o padrão, na qualidade (primitiva) que denominamos comprimento. $\mathrm{O}$ número que emerge da comparação é uma medida da grandeza física que introduzimos e denominamos distância.

É importante ressaltar que, por definição, a medida dita exata do tamanho de um objeto não existe, uma vez que devido a definição do procedimento de medida, sempre haverá uma barra de erros, que traduz o refinamento da escala, que também por definição leva a um procedimento sem fim de refinamento [36]. Utilizando a noção de direção e de eixos reais, o conceito de espaço, enquanto elemento geométrico, fica definido. Ou seja, a partir de uma variedade $\mathbb{R}^{3}$, introduz-se o locus de cada sistema físico na relação estática com os demais. A estrutura intrínseca dessa variedade é a de espaço vetorial; e outros elementos geométricos podem ser incorporados a esta estrutura, mas isso irá depender do experimento.

Dependendo do tipo de relação, a conformação entre os objetos físicos mudam. Essa mudança é denominada movimento, que se estabelece também como um conceito primitivo. O quanto e quão rápido os sistemas mudam uns com relação aos outros é definido a partir da construção de um padrão não estático, que é escolhido como por possuir um movimento repetitivo . Da comparação dessa repetição com outros sistemas em movimento emerge um número Real, $\mathbb{R}^{1}$, que é uma medida da grandeza física chamada tempo. A repetição do padrão é assumida $a$ priori. O teste da sua precisão requer a construção de outro padrão - outro sistema em movimento que se repete mais rapidamente que o primeiro pode ser assim utilizado. O padrão, o sistema físico que se repete, é denominado relógio, e a unidade da repetição é chamada período de tempo. A história da ciência descreve com acúrácia a introdução e utilidade desses padrões, desde o movimento do Sol, da Lua e das estrelas, até o relógio mecânico, como descrito na seção anterior. Aqui é importante apenas destacar que a física utiliza o mesmo método de definição de padrão e escala para introduzir as grandezas tempo e espaço.

Essa concepção realista com que a física opera não introduz um objeto ou ente chamado tempo em si, ou tempo absoluto (o mesmo vale para o espaço). Embora esse atributo de em si seja tão antigo quanto os gregos, 
que antropomorfizaram o tempo no mito de Cronos, na física esse conceito não é utilizado. Newton, nos Principia, para construir a sua axiomatização da mecânica, inicia elaborando sobre o conceito de tempo absoluto (o tempo em si) e do tempo relativo (o tempo definido através de processos - relógios). Somente nesses parágrafos iniciais, é que os Principia abordam esse conceito de tempo absoluto, uma herança da filosofia e da teologia, como apontado também na seção anterior. Newton utilizará o tempo relativo em suas contas. E é esse legado que a práxis da física adotou desde então, a despeito da intensa discusão que se sucedeu com o advento da teoria da relatividade. Neste caso, muitos físicos quase que sucumbiram a uma inclinação irresistível de se referir ao tempo como um objeto em si, e não como um conceito definido a partir de procedimentos de medida - com os relógios. Todavia, no ato de perfazer suas contas e estabelecer suas previsões, os físicos continuaram a proceder, independente das crenças e concepções filosóficas, como Newton, utilizando o tempo assim como o espaço definidos pelo conceito primitivo de relações (ou processos) entre objetos físicos.

Com a noção de tempo mapeada na reta real $\mathbb{R}$ e do comprimento e dimensões mapeados no $\mathbb{R}^{3}$, chega-se a uma variedade $\mathbb{R}^{4}=\mathbb{R}^{3} \otimes \mathbb{R}^{1}$, que serve de substrato aos eventos físicos. Pontos nesta variedade estabelecerão os sistemas de referências inerciais (SI's), definidos pelas regras de transformações desses pontos, de um a outro, de modo a se considerar invariantes as leis da física. Essas regras de transformações, que necessariamente precisam ser estabelecidas/satisfeitas pelo experimento, são chamadas de simetrias do espaço-tempo, e sua estrutura matemática é em muitos casos, mas não em geral, a de um grupo de Lie. Na física não-relativística a definição dos SI's é dada pelo grupo de Galilei e na física relativísitica, é o grupo de Lorentz (ver a próxima seção). Há ainda outros grupos de simetrias no espaço-tempo, como o grupo conforme e o de De Sitter, cada qual a tratar de aspectos específicos dos sistemas físicos (mecãnicos).

Dessa análise, alguns pontos merecem, em resumo, destaque. Primeiro, o elemento ontológico (primitivo) que a física utiliza para definir suas grandezas estruturantes é a noção de relação entre dois ou mais sistemas físicos, que pode ser de natureza estática (a conformação entre os sistemas não muda, e que leva ao conceito de espaço) e não-estática (a conformação muda, o que se chama de movimento, e leva à noção de tempo). Com esses dois elementos, tempo e espaço ficam definidos a partir do processo de construção de padrões e mensuração; e assim outras grandezas podem ser introduzidas para se analisar a natureza do movimento. Esse é o caso da velocidade, definida como variações do espaço e no tempo. O outro aspecto da análise é que a caracterização de tempo e espaço, a partir dessa práxis da física, descarta, por não fazer uso, o conceito de tempo absoluto, ou tempo em si, enquanto um ente. A física abandona essa discussão, e a partir dessa postura, se aproxima mais da sociologia [51], que da filosofia, com suas metafísicas transcedentes ou não. Isso é um fato, pois a introdução do conceito de tempo pela práxis da física sempre se refletiu em uma demanda social para organizar as atividades de agrupamentos humanos, enquanto elaborava uma visão de conjunto do mundo. O aspecto interessante desse procedimento é que a noção de tempo e de espaço (por não serem absolutos) podem receber novos atributos; isto é o que aconteceu com a postulação da teoria da relatividade restrita. Este aspecto será detalhado mais adiante. Antes, a caracterização dos sistemas mecânicos deve ser elaborada.

\section{Sistemas mecânicos: ponto inercial (material) e campo}

Com o desenvolvimento da síntese de Newton nos Principia, os sistemas mecânicos passaram a ser classificados em dois tipos: uns descritos por pontos inerciais (ou materiais) e outros por campos. Nesta seção uma análise desses dois conceitos será conduzida com ênfase em aspectos que evidenciam elementos constitutivos de um projeto mecânico geral. Não será apresentado uma descrição histórica desses conceitos, em especial da noção de campo, o que pode ser encontrado em ótimas referências na literatura [52,53]. A revisão será conceitual e atual, mas tanto quanto possível pedagógica, ressaltando aspectos que escapam em grande medida aos textos didáticos. Por isso alguns elementos básicos das formulações usuais da mecânica serão repetidos.

\subsection{Dinâmica do ponto material}

Um ponto material, conceito introduzido por Newton, é um sistema físico definido como um ponto geométrico um conceito primitivo -, com atributos mecânicos, como inércia (massa), carga elétrica, spin, etc. (Todas essas grandezas são definidas de modo preciso a partir de um aparato experimental.) Um sistema assim é também chamado de partícula e sua principal característica é a localidade. Sistemas compostos de muitos pontos materiais são chamados de sistemas de muitas partículas, ou sistemas de muitos corpos. Um exemplo desse tipo é o sistema solar, em que os planetas e o Sol são considerados pontos materiais interagindo entre si pela ação da força gravitacional. Do mesmo modo, um gás pode ser considerado como um sistema de muitas partículas.

Um sistema de muitas partículas apresenta caracterísitcas próprias da coletividade. Por exemplo, em uma rede cristalina, na qual cada sítio contém uma partícula (átomo), que pode interagir com os demais vizinhos, ocorrem os chamados modos de vibração, que refletem formas de movimento individual, mas associadas à coletividade dessas partículas. Isso leva ao conceito, tipicamente coletivo, de fônon. Entretanto, há ainda outras, mas não menos fundamentais, características associadas a coletividade, como as propriedades térmicas dessa coletividade. Essas propriedades, por vez, precisam ser compatíveis com as leis da termodinâmica, o que conduz a problemas ainda não completamente resolvidos no âmbito da física, como a reversibilidade microscópica em contraposição 
com a irreversibilidade macroscópica, caracterizada pela segunda lei da termodinâmica, ou lei de crescimento da entropia. Este aspecto não será abordado no presente trabalho. Por ora, é necessário detalhar um pouco mais o conceito de ponto material, para contrastá-lo, na sequência, com o conceito de campo.

Se para a localização, conformação e orientação no espaço forem necessários pelo menos $N$ variáveis, então dizemos que o sistema possui $N$ graus de liberdade. As $N$ variáveis , $q=\left(q_{1}, \ldots, q_{N}\right)$, são chamadas de $N$ coordenadas generalizadas. Este conceito de localização do sistema mecânico no espaço é fundamental para descrever a condição de movimento do sistema em questão; mas não é exaurível pois não traz informação adicional sobre a possíbilidade do sistema se movimentar e estar em outro lugar no instante seguinte. Essa demanda leva a definição de "estado mecânico" do sistema, que pode ser introduzido como segue.

Definição 1. Estado mecânico. O Estado mecânico de um sistema físico em um instante de tempo $t$, denotado por $\mathcal{E}(t)$, é definido por um conjunto de informações sobre o sistema que estabeleça sua configuração espacial e apresente informações sobre a natureza do movimento do sistema. Este conceito de estado mecânico fica completamente estabelecido a partir da experimentação; e esta deve prover uma classificação dos sistemas mecânicos (quer sejam campos ou pontos materiais), em classes específicas de estados mecânicos.

Talvez o mais simples caso seja aquele dado pelas coordenadas generalizadas e as respectivas velocidades generalizadas, isto é as derivadas temporais. Formalmente isto é escrito como o seguinte conjunto de variáveis: $\mathcal{E}(t)=(q, \dot{q}), \operatorname{com} q=\left(q_{1}, \ldots, q_{N}\right), \dot{q}=\left(\dot{q}_{1}, \ldots, \dot{q}_{N}\right)$, where $\dot{q}_{i}=d q_{i} / d t$. Assim o estado mecânico de um sistema de $N$-pontos materiais, $N$-partículas, fica definido pela posição - localização espacial -, e pela velocidade, uma informação local sobre o movimento do sistema. Esta definição deve respeitar as limitações experimentais, de modo que $q(t)$ and $\dot{q}(t)$ possam ser mensuradas no mesmo instante de tempo na mesma barra de erro da mensuração. Observe que na definição de estado, a posição e a velocidade são variáveis independentes, e este conjunto de informações descreve então o que o sistema mecânico é do ponto de vista do movimento.

$\mathrm{O}$ estado mecânico definido em termos de posição e velocidade é suficiente para tratar uma classe ampla de sistemas mecânicos. Entretanto, há aqueles em que a caracterização necessita ser dada pela posição e derivadas mais altas, como $\{q, \dot{q}, \ddot{q}\}$. Esse tipo de sistema não será considerado aqui.

Para que se possa descrever o movimento dos sistemas físicos, é necessária a especificação de um princípio geral que trate da evolução temporal do estado. Assim se adota o seguinte princípio:

Axioma 1. Causalidade na evolução do estado mecânico. Considerando a evolução do estado mecânico de um sistema físico entre dois instantes de tempo, $\mathcal{E}\left(t_{i}\right)$ e $\mathcal{E}\left(t_{f}\right)$, onde $t_{i}$ é um instante inicial, e $t_{f}$ é um instante final, assume-se que há uma relação de causalidade entre os dois estados mecânicos do sistema, de modo a garantir que repetidas as mesmas condições iniciais, o estado do sistema, e portanto o sistema físico, evoluirá do mesmo modo.

Com este princípio de causalidade, na situação em que $t_{f}=t_{i}+\delta t$ com $\delta t \ll 1$, pode-se esperar que o estado evolua condicionado a uma equação diferencial no tempo. Para discutir a natureza dessa relação é necessária a definição dos sistemas físicos especificos. Ou seja, devemos definir aquilo que na mecânica irá representar cada sistema físico, diferenciando assim um do outro.

Definição 2. Sistemas físicos específicos - Lagrangiana. A especificação de cada sistema mecânico é dada por uma função escalar do estado do sistema; isto é, uma função invariante por transformações de um SI a outro. Esta função é denominada Lagrangiana $(\mathcal{L}=\mathcal{L}(\mathcal{E}))$.

O fato de a Lagrangiana ser um escalar garante que o sistema em análise, além de invariante, seja o mesmo para dois quaisquer observadores localizados em distintos SI's. Isto significa que a Lagrangiana será um escalar pelas transformações no espaço-tempo. Para a situação de pontos materias, a Lagrangiana é escrita como $\mathcal{L}(q, \dot{q})=$ $\mathcal{L}\left(q_{1}, \ldots, q_{N}, \dot{q}_{1}, \ldots, \dot{q}_{N}\right)$.

Um determinado sistema evolui de um instante de tempo $t_{i}$, na posição $q\left(t_{i}\right)$, para um instante de tempo $t_{f}$, na posição $q\left(t_{f}\right)$, seguindo uma única trajetória no espaço de configurações, ou seja uma única sequência de pontos entre $q\left(t_{i}\right) \rightarrow q\left(t_{f}\right)$, dentre uma infinidade de possibilidades de funções do tempo ligando $q\left(t_{i}\right)$ a $q\left(t_{f}\right)$. O problema mecânico apresentado deste modo, naturalmente aponta para a construção de um princípio de extremo. Assim procura-se um funcional no tempo, envolvendo o intervalo entre $t_{i}$ e $t_{f}$, a partir de $\mathcal{L}(q, \dot{q})$. A escolha mais simples é um funcional linear do tipo

$$
\mathcal{A}=\int_{t_{i}}^{t_{f}} \mathcal{L}(q, \dot{q}) d t,
$$

que também deve ser invariante pelas transformações do espaço-tempo.

A especificação do caminho físico é dada pela condição de extremo $\delta A=0$, com as coordenadas $q\left(t_{i}\right)$ e $q\left(t_{f}\right)$ fixadas, i.é, $\delta q\left(t_{1}\right)=\delta q\left(t_{2}\right)=0$. Isto conduz às equações de Euler-Lagrange,

$$
\frac{d}{d t} \frac{\partial \mathcal{L}}{\partial \dot{q}_{i}}=\frac{\partial \mathcal{L}}{\partial q_{i}} \quad ; i=1, \ldots, N
$$

que são equações diferenciais ordinárias de segunda ordem no tempo.

Esta análise está conduzida de modo geral, ou seja, sem a especificação da estrutura de simetria do espaçotempo. Vamos escolher inicialmente o grupo de Galilei, definido pelo seguinte conjunto de transformações do 
espaço-tempo 54,55 . 'Dois sitemas inerciais, $S$ e $\bar{S}$, são Galileanos, se os fenômenos físicos analisados em $S$ e em $\bar{S}$ estiverem relacionados pelas seguintes transformações,

$$
\begin{aligned}
\overline{\mathbf{x}} & =R \mathbf{x}+\mathbf{v} t+\mathbf{a}, \\
\bar{t} & =t+b,
\end{aligned}
$$

onde $q \equiv \mathbf{x}$ tal que $\overline{\mathbf{x}}=(\bar{x}, \bar{y}, \bar{z})$ e $\bar{t}$, descrevem em $\bar{S}$, respectivamente, um vetor de localização no espaço Euclidiano e uma coordenada no tempo. Estas transformações são caracterizadas por 10 parâmetros: 3 associados às rotações, $R$; 3 parâmetros associados às transformações dos sistemas de coordenadas descrevendo a velocidade v, de um sistema $\bar{S}$ com relação ao outro $S$ - os três parâmetros são as três componentes de $\mathbf{v} ; 3$ parâmetros associatrados às translações no $\mathbb{R}^{3}$, ou seja as três componentes de $\mathbf{a}$; e um (1) parâmetro, $b$, associado à diferença de tempo entre um relógio em $\bar{S}$ e outro em $S$. Isso significa que o tempo transcorre da mesma forma nos dois referenciais. O conjunto de transformações dadas pelas Eqs. (3) e (4) descreve o grupo de Galilei.

Essas transformações podem então ser utilizadas na modelagem de sistemas físicos, considerando que a Lagrangiana $\mathcal{L}$ é um escalar perante transformações do grupo de Galilei. Isto está apresentado com certo detalhe tanto no livro de Landau e Lifshitz [54], como no livro de Sudarshan e Mukunda 55. Uma classe geral, mas não única, dessas funções de Lagrange é dada por

$$
\mathcal{L}=T\left(\dot{q}_{1}, \ldots, \dot{q}_{N}\right)-V\left(q_{1}, \ldots, q_{N}\right)=T(\dot{q})-V(q),
$$

onde o segundo termo do lado direito, $V(q)$, pode expressar a interação das partículas entre si e dessas com campos externos. Quando a interação se anula, a função de Lagrange fica, então, dependente somente da velocidade. Para um sistema de $N$ graus de liberdade, $T$ é dada por

$$
T=\sum_{i=1}^{N} \frac{1}{2} m_{i} \dot{q}_{i}^{2}
$$

e assim

$$
\mathcal{L}=\sum_{i=1}^{N} \frac{m_{i}}{2} \dot{q}_{i}^{2}-V\left(q_{1}, \ldots, q_{N}\right) .
$$

Usando a Eq. (2), obtém-se a segunda lei de Newton

$$
m_{i} \frac{d^{2} q_{i}}{d t^{2}}=-\frac{\partial V}{\partial q_{i}}
$$

na qual $F_{i}=-\partial V / \partial q_{i}$ é a i-ésima componente da força atuando sobre a partícula localizada pelas coordenadas $q_{i}$. Aqui, este resultado corresponde a comprovação experimental.

\subsection{Invariantes de movimento: teorema de Amalie Emmy Noether}

As equações (2) são de segunda ordem na derivada temporal, e, portanto, requerem um conjunto de $2 N$ constantes para que a solução fique unicamente especificada. Isto motiva a procura de constantes gerais de movimento, que são escritas em termos do estado mecânico do sistema. No caso, são escritas como $C\left(q_{1}, \ldots, \dot{q}_{N} ; t\right)$; e assim $C$ não varia com o tempo, a despeito de $q_{1}, \ldots$ e $\dot{q}_{N}$ mudarem ao longo do trajeto evolutivo do sistema. Mas, após a solução das $N$ equações de Euler-Lagrange, podemos explicitar as $2 N$ constantes de integração (independentes), como funções de $q, \dot{q}$ e $t$; isto é 54

$$
\begin{gathered}
C_{1}=C_{1}\left(q_{1}, \ldots, \dot{q}_{N} ; t\right) \\
\vdots \\
C_{2 N}=C_{2 N}\left(q_{1}, \ldots, \dot{q}_{2 N} ; t\right)
\end{gathered}
$$

Encontrar as variáveis mecânicas constantes representa, então, deduzir a própria solução do problema mecânico. De fato, desde que possamos encontrar $2 N$ constantes de movimento, podemos invertê-las com relação a $q(t)$ e $\dot{q}(t)$, e encontrar a trajetória do sistema.

Um procedimento geral para encontrar invariantes é através do teorema de Noether, que especifica uma constante de movimento a uma simetria do espaço-tempo. A partir disso, se constrói diversas variáveis cinemáticas, como carga, massa, momentum, energia, entre outras. Aqui é importante discutir, mesmo que de modo breve, o conceito de energia, E, o invariante associado a homogeneidade temporal. Isso significa que a Lagrangiana não depende explicitamente do tempo. Este fato juntamente com as equações de Euler-Lagrange conduz a seguinte expressão para $E$ [54,

$$
E=\sum_{i=1}^{N} \frac{m_{i}}{2} \dot{q}_{i}^{2}+V\left(q_{1}, \ldots, q_{N}\right) .
$$

Nos livros textos básicos, usualmente, se introduz o conceito de energia associado ao de trabalho mecânico, grandeza que representa uma medida de esforço para um agente externo alterar o estado de movimento do sistema mecânico. Esse tipo de grandeza é fundamental para se introduzir a noção de rendimetno de uma máquina. Devido ao teorema de conservação, em muitas situações induz-se o estudante a pensar na energia como "algo substancializado", e a linguagem coloquial se apropria pelo menos parcialmente desse entendimento. Quando se fala, "em um choque de dois corpos há transferência de energia", isso só significa que os corpos terão seus estados mecânicos alterados devido a interação. Na linguagem de Newton, isso ocorre respeitando a terceira lei, a de ação e reação.

É importante ressaltar que o conceito de energia é, mecanicamente, bem definido e, principalmente, é uma quantidade mensurável. A conservação, na mecânica, é resultado do teorema de Noether. Com o advento da termodinâmica, a conservação da energia passa a ser considerada como uma lei (e não um teorema), garantida pelos experimento de Joule 56,57. Outro aspecto a ser salientado é que o conjunto das variáveis mecânicas é estabelecido a partir das quantidade que introduzem o estado mecânico. Este é o caso de variáveis como energia, momentum, entre outras, escritas como funções da 
posição e velocidade. Estas variáveis básicas, definindo o estado, são chamadas assim de variáveis canônicas.

O conjunto dessas variáveis, axiomas e definições, introduzido até aqui e associado aos sistemas físicos descritos como pontos materiais, pode ser estendido para tratar então um outro tipo de sistema mecânico: os campos.

\subsection{Dinâmica de um campo}

Um campo é um sistema mecânico definido como um atributo físico a pontos do espaço-tempo, contendo características do movimento (mecânicas), como inércia, energia, momento, carga, etc. Um elemento importante na caracterização de um campo é a não localidade e sua não redutibilidade a pontos inerciais (materiais). Um campo pode ser representado matematicamente como uma função de pontos do espaço e tempo, isto é, $f=f(\mathbf{x}, t)$, onde $f$ expressa a quantidade física, a ser bem definida e de acesso experimental. A natureza matemática de $f$ fica estabelecida pelo experimento, e dependendo do que descreve e da estrutura do espaço-tempo pode ser um escalar, um vetor, um tensor ou ainda um espinor. Alguns casos ajudam a esclarecer a definição.

Um exemplo paradigmático é o campo de velocidades em um fluído, usualmente denotado por $\mathbf{V}(\mathbf{x} ; t)$, onde $\mathbf{V}$ é o atributo físico (no caso o vetor velocidade) dos pontos do espaço-tempo $(\mathbf{x} ; t)$. Como a propriedade $\mathbf{V}$ muda com o tempo, é possível estabelecer-lhe as equações de movimento, através de um princípio de extremo, generalizando o caso de um ponto material. A dinâmica é dada pela equação de Navier-Stokes. Outro exemplo é uma onda se propagando em uma corda. A onda é descrita como $Y(x, t)$, onde $Y$ representa a altura no eixo $y$ do ponto $x$ da corda, em certo instante de tempo t. A equação de movimento para $Y(x, t)$ é a conhecida equação da onda.

O fenômeno eletromagético, descrito pelo campo elétrico, $\mathbf{E}(\mathbf{x} ; t)$, e o campo magnético, $\mathbf{B}(\mathbf{x} ; t)$, é um outro exemplo conhecido de um campo, para o qual a evolução temporal é dada pelas equações de Maxwell. De modo covariante, escreve-se o tensor de campo $F^{\mu \nu}(x)$, onde $x$ é um quadrivetor fornecendo uma posição do espaçotempo de Minkowski e $\mu, \nu=0,1,2,3$. As componentes de $F^{\mu \nu}(x)$ estão relacionadas com as componentes de $\mathbf{E}(\mathbf{x} ; t)$ e $\mathbf{B}(\mathbf{x} ; t)$ [58]. Neste caso a Lagrangiana é um escalar de Lorentz.

Em mecânica quântica, a amplitude de probabilidade, $\psi(\mathbf{x} ; t)$, é um outro exemplo de campo, de natureza escalar pelas transformações de Galilei, e que se associa à densidade de probabilidade dada por $|\psi(\mathbf{x} ; t)|^{2}$. Isto define completamente o significado físico de $\psi(\mathbf{x} ; t)$, inclusive experimentalmente, enquanto um campo ligado ao conceito de probabilidade, com características físicas de inércia (massa) e com uma dinâmica descrita pela equação de Schrödinger. As funções $\psi(\mathbf{x} ; t)$ descrevem as condição de movimento de objetos mecânicos chamados "partículas não-relativísticas". A evolução espaço-temporal de $\psi$ fornece o movimento das partículas, caracterizadas, como no caso de pontos materiais, por suas características me- cânicas, como massa, momentum, carga, entre outras. Ou seja, a denominação "partícula" expressa as características mecânicas descritas pelo campo. Para sistemas quanto-mecânicos, o campo $\psi(\mathbf{x} ; t)$ está definido no espaço de Hilbert; mas a descrição mais geral e completa de um sistema quântico é obtida quando a representação tem como ponto de partida o espaço de Fock. Isso será válido também para o campo eletromagnético, que leva à chamada eletrodinâmica quântica.

Observe que $\psi(\mathbf{x} ; t)$ e $F^{\mu \nu}(x)$, por exemplo, são nãolocais, e não-redutíveis a pontos materiais, por definição. A denominação "partícula", aqui empregada, para descrever por exemplo o fóton, não significa que o objeto físico, fóton, seja uma "bolinha" que em princípio possa ser tratada por um ponto (no sentido geométrico). A denominação "partícula" expressa um ente fundamental, até pelo menos o experimento apontar que possui estrutura. Isto é o que aconteceu com o próton, inicialmente considerado como uma partícula elementar e descrita pelo campo de Dirac. Depois, experimentalmente, observou-se que o próton possuía estrutura. De um ponto de vista teórico-mecânico, isso significa que o campo de Dirac, que descrevia algumas das propriedades do próton, precisou ser considerado como uma combinação de outros campos interagentes; ou ainda, os campos que descrevem os quarks e os campos que descrevem os glúons (as partículas que compõem os prótons). Ressalte-se: tudo isso localizado em dimensões da ordem de $10^{-15} \mathrm{~m}$; mas ainda assim são campos, entes que não se reduzem a pontos inerciais.

Para encontrar a descrição mecânica de um campo, procedimentos introduzidos para o caso de pontos materiais são então generalizados. Assim como um ponto material, $\psi(\mathbf{x} ; t)$, o campo de Schrödinger, descreve a conformação do sistema físico no espaço-tempo. A informação pertinente a sua mudança espaço-temporal provém das derivadas de $\psi(\mathbf{x} ; t)$. O estado mecânico do campo de Schrödinger é então dado por $\psi(\mathbf{x} ; t)$ e por suas derivadas. Estas quantidades definem, em paralelo ao caso de pontos inerciais, o estado mecânico de campos clássicos. Este procedimento será válido para outros campos.

Considere então generalizar $q(t)$, enquanto uma função de um parâmetro real, $t$, para uma função de $r+1$ parâmetros dada por $q(\tau)=q\left(\tau^{0}, \tau^{1}, \ldots ., \tau^{r}\right)$, de tal modo que a (densidade de) Lagrangiana fica dada por $\mathcal{L}(q, \partial q)=\mathcal{L}\left(q, \partial_{0} q, \partial_{1} q, \ldots, \partial_{r} q\right)$, onde $\partial_{\alpha} q=\partial q / \partial \tau^{\alpha} . \mathrm{A}$ ação fica escrita como

$$
\mathcal{A}=\int_{\gamma} d^{r} \tau \mathcal{L}(q, \partial q),
$$

onde $\gamma$ é uma hiper-superfície de integração. Considera-se também que em $\gamma,\left.\delta q(\tau)\right|_{\gamma}=0$, e calculemos $\delta \mathcal{A}=0$. Isto leva a equação de Euler-Lagrange,

$$
\partial_{\alpha} \frac{\partial \mathcal{L}}{\partial\left(\partial_{\alpha} q\right)}=\frac{\partial \mathcal{L}}{\partial q} ; \quad \alpha=0,1, \ldots, r .
$$

Aqui, a regra da soma sobre os índices repetidos está sendo usada. Se $q(\tau)$ possui mais do que uma componente, 
então devemos substituir $q$ por $q_{j}(\tau)$, com $j=1,2, \ldots, N$, com $N$ o número máximo de componentes independentes de $q(\tau)$. Há assim $N$ equações de Euler-Lagrange [27].

Este resultado é puramente teórico. A interpretação física provém da especificação de $\tau$ e do conteúdo físico de $q(\tau)$. Definiremos $\tau$ como um 4-vetor no espaço-tempo de Minkowski, $\mathbb{M}(3,1)$, com $\tau^{0} \equiv x^{0}=c t ; \tau^{1} \equiv x^{1}$, $\tau^{2} \equiv x^{2}, \tau^{3} \equiv x^{3}$, isto é $\tau=x=\left(x^{0}, x^{1}, x^{2}, x^{3}\right)$. A métrica é dada por $g=\left(g^{\mu \nu}\right)$ que é uma matriz diagonal, tal que diag $g=(1,-1,-1,-1,-1)$, com $g=g^{-1}$; tal que $\left(g^{\mu \nu}\right)^{-1} \equiv g_{\mu \nu}, x_{\mu}=g_{\mu \nu} x^{\nu} \quad$ e $x^{\mu}=g^{\mu \nu} x_{\nu}$ [17. A coordenada generalizada, $q(x)$, é então um campo especificado ao se fornecer a qualidade física de cada ponto do espaço-tempo. Considerando um campo escalar, é usual mudar a notação e escrever $q(\tau) \rightarrow \phi(x)=\phi(t, \mathbf{x})$, que fornece a configuração espaço-temporal do campo. A equação de Euler-Lagrange, Eq. (9), fica escrita como

$$
\partial_{\alpha} \frac{\partial \mathcal{L}}{\partial\left(\partial_{\alpha} \phi\right)}=\frac{\partial \mathcal{L}}{\partial \phi},
$$

com $\alpha=0,1,2,3$. A densidade de Lagrangiana $\mathcal{L}(\phi, \partial \phi)$, assim como $\phi$, é um escalar por transformações de Lorentz, contruídas a partir de transformações lineares em $\mathbb{M}(3,1)$; ou seja, transformações que deixam invariante o produto escalar entre dois 4-vetores; isto é, $x \cdot y=g_{\mu \nu} x^{\nu} x^{\mu}$ é invariante.

A transformação linear é escrita como

$$
x^{\prime \mu}=\Lambda_{\nu}^{\mu} x^{\nu}
$$

de modo que $\Lambda_{\nu}^{\mu}=\partial x^{\mu} / \partial x^{\nu}$. Além disso, a invariância do produto escalar conduz à invariância da métrica: $\Lambda^{T} g \Lambda=g$, onde $\Lambda^{T}$ é a matrix transposta de $\Lambda$. Disto segue que $\operatorname{det} \Lambda= \pm 1$. Para $\operatorname{det} \Lambda=1$, as denominadas transformações próprias de Lorentz, tem-se um grupo de Lie conectado a identidade. Neste caso, considerando transformações infinitesimais, segue

$$
x^{\prime \mu}=x^{\mu}+\omega_{\nu}^{\mu} x^{\nu},
$$

onde $\omega_{\rho \nu}=g_{\rho \mu} \omega^{\mu}{ }_{\nu}$ é uma matriz antissimétrica. Então, as transformações de Lorentz, atuando em uma representação infinito-dimensional, em outras palavras, atuando em um espaço de funções analíticas definidas em $\mathbb{M}(3,1)$ ), são dadas por

$$
\Lambda=\exp \left[\omega_{\mu \nu} M^{\mu \nu}\right]
$$

onde $M^{\mu \nu}$, os geradores de transformações de Lorentz, são escritos como $M^{\mu \nu}=i\left(x^{\mu} \partial^{\nu}-x^{\nu} \partial^{\mu}\right)$.

Vários modelos especificados por densidades de Lagrangiana invariantes por Lorentz podem ser então construídos. Um dentre os mais simples tem por densidade de Lagrangiana

$$
\mathcal{L}=\frac{1}{2} \partial_{\alpha} \phi \partial^{\alpha} \phi-\frac{1}{2} m^{2} \phi^{2}+J \phi,
$$

de modo que, substituindo nas equações de Euler-Lagrange, Eq. (10), segue

$$
\partial_{\alpha} \partial^{\alpha} \phi+m^{2} \phi=J
$$

ou seja, a equação de Klein-Gordon, descrevendo uma partícula quântica relativística de massa $m$, em um termo de fonte $J$. Os detalhes da construção de um modelo como este (entre outros como para o campo eletromagnético e o de Schrödinger) não serão abordados aqui, e podem ser encontrado em outros lugares [21]. Contudo, é importante finalizar esta seção retornando a pontos fundamentais para a conceitualização física de um campo e de um sistema de pontos inerciais.

Em resumo, há duas possibilidades para se tratar um sistema físico: pontos inerciais e campos. Existe ainda uma terceira possibilidade que é considerar sistemas híbridos de campos e pontos inerciais. Essas possibilidades estabelecem o problema de se escolher a forma de tratar um evento físico. Por exemplo, um gás pode ser considerado como um fluido, neste caso descrito por campos; mas também pode ser tratado como um conjunto de pontos inerciais interagindo entre si. Mais uma vez, aqui, é a práxis da física, através do experimento, quem ditará a forma mais adequada de descrição. Toda a física contemporânea, representada através do modelo padrão de partículas ou o cosmológico, assim como toda a mecânica quântica não-relativística, tratando da física da matéria condensada, modela os fenômenos do movimento através do conceito de campo. Mas a física não tenta, e não pode tentar, estabelecer que o mundo seja ontologicamente composto de campos. A modelagem da física atual usa o conceito de campo, respeitando a barra de erros dos experimentos: isso leva a 62 partículas fundamentais na natureza, além do gráviton. Contudo, não há inferências, em princípio, para escalas inacessíveis; e assim, a descrição dos fenômenos através de pontos inerciais pode vir a prevalecer em outros contextos mais gerais. Essa observação significa que o compromisso (quase que atávido) da física é com os resultados experimentais, estes vistos como uma espécie de bússola, a apontar a direção de se estabelecer, cada vez mais precisa, uma visão de conjunto dos fenômenos observados, onde o limite da observação é o refinamento das escalas dos instrumentos de medida. Em outro dito, a física não trata de afirmar ou negar se a natureza do mundo físico é de campos ou partículas. O elemento ontológico básico da física é o ponto de partida para estabelecer os processos de mensuração; ou seja, as relações entre os objetos físicos. Com essa premissa, primitiva conceitualmente, constrói-se teorias que terão a validade estabelecida pelas barras de erro dos experimentos. Ou seja, à física, por construção, não está dada a possíbilidade de estabelecer teorias ou afirmações últimas sobre o movimento. Mas então se pode procurar os elementos básicos para a construção das teorias mecânicas, e assim compreender a diferença e limitações conceituais entre formulações distintas.

\section{Elementos das teorias do movimento}

Os elementos para um programa mecânico são apresentados nesta seção, com o objetivo de sintetizar a análise das seções anteriores, que resulta em um desenvolvimento pedagógico de resultados previamente desenvolvidos 31,35], 
mas indo além, por evidenciar o procedimento experimental. Exploramos, a propósito de aplicação, a análise do conceito de tempo na física relativística e não-relativística. $\mathrm{Na}$ próxima seção avaliaremos a teoria cinética relativística.

\subsection{Elementos constitutivos de uma mecânica}

Um programa mecânico (geral) pode ser definido a partir de cinco elementos constitutivos, dados como segue.

e1 - Assume-se como conceito primitivo a existência do espaço e do tempo descrito por uma variedade $\mathcal{V}=\{\mathbf{x}, t\}$, com $\mathbf{x}$ designando vetores do $\mathbb{R}^{3}$, e $t \in \mathbb{R}$, o tempo. As quantidades $\mathbf{x}$ e $t$ possuem suas propriedades especificadas através de processos físicos. Ou seja, é a mensuração via processos, que estabelece o tempo e o espaço. A mensuração das propriedades dos sistemas físicos é implementadada a partir de um locus no epaço e tempo, a ser denotado por $S$ e chamado de sistema de referência inercial. Os sistemas de referência são definidos pela especificação da conexão entre si. Esta associação entre dois sistemas, $S$ e $\bar{S}$, se estabelece por um mapeamento $\mathfrak{G}: S \rightarrow \bar{S}$, sendo $\mathfrak{G}$ transformações especificadas pelo experimento. Uma situação geral é que $\mathfrak{G}$ seja um um grupo de simetria em $\mathcal{V}$, de tal modo que os fenômenos físicos em $S$ e $\bar{S}$ são descritos matematicamente da mesma maneira. Mas pode ocorrer de ser um semi grupo, e assim contemplar processos irreversíveis. Num caso ou noutro, será o experimento quem estabelecerá a natureza de $\mathfrak{G}$.

e2 - Assume-se como conceito primitivo a existência de um conjunto de variáveis mecânicas denotado por $\mathcal{D}=\{a, b, c, .$.$\} , entre as quais estão aquelas$ que descrevem a localização, velocidade, momentum, energia, dentre outras. Um conjunto básico dessas variáveis, a partir das quais todas as outras são construídas, é denominado de observáveis ou variáveis canônicas. Essas serão os observáveis do sitema e minimamente possuirão a estrutura de espaço linear.

e3 - Assume-se como conceito primitivo a existência de sistema físico. Um sistema físico fica descrito, e é mecanicamente caracterizado, pela noção de estado mecânico $(\mathcal{E})$ definido de tal forma a: (i) estabelecer a localização do sistema no espaço e no tempo, e (ii) prover elementos do movimento; isto é, fornecer uma ou mais característica intrínsica do movimento do sistema escolhida como um conceito primitivo. O estado descreverá sistemas mecânicos de dois tipos, considerados também como conceitos primitivos: (a) aqueles compostos de pontos inerciais (ou materiais), entes mecânicos cujo estado é especificado por grandezas locais no espaço e no tempo, e (b) campos, objetos mecânicos cujo estado é descrito por grandezas que são funções em todo $\mathcal{V}$, e a priori não são redutíveis à pontos inerciais. (iii) Dado um observável $\mathcal{O}$, deve existir uma regra a ser estabelecida experimentalmente de modo que $\mathcal{E}(\mathcal{O}) \in \mathbb{R}^{n}$, correspondendo ao processo de mensuração.

e4 - Os sistemas mecânicos específicos são definidos por uma função do estado mecânico, chamada Lagrangiana. Esta função é um escalar, por tranformações envolvendo a mudança dos sistemas de referências, ou seja por $\mathfrak{G}$, assegurando assim que todos os observadores estarão descrevendo o mesmo sistema físico.

e5 - Assume-se a existência de uma lei causal conectando os diversos estados do sistema; lei esta a ser invariante por $\mathfrak{G}$. A lei causal pode ser encontrada por um princípio de extremo compatível com $e_{1}$.

Neste ponto, a caracterização do tempo a partir de relações entre objetos físicos deve ser detalhada. (Análise similar pode ser conduzida para o espaço.) Enquanto processo podemos então ter em mente as oscilações de um pêndulo, e como sistema físico, uma partícula descrita como um ponto material. Vamos denominar por $\mathcal{P}$ o conjunto de elementos que caracterizam os processos, $\mathcal{P}=\{P \in \mathbb{R}\}$. Os $P$ são as oscilações, no caso do pêndulo, ao se comparar o oscilador e o movimento de uma partícula de um ponto a outro no espaço. Esta comparação, que se dá por uma relação entre os dois objetos, tida como um conceito primitivo, gera um número $P$, que será o valor associado a uma gradeza física a ser denominada "tempo", t. Assim se pode estabelecer uma aplicação, $\mathfrak{n}: \mathbb{R} \rightarrow \mathbb{R}$, entre cada elemento $P$ e $t \in \mathbb{R}$. Nesse caso, $t$ é especificado pela reta real, ou seja, é uma coordenada, em que cada valor de $t$ é chamado de instante de tempo. A aplicação $\mathfrak{n}$ é definida como sendo linear (embora outras possibilidades sejam possíveis), isto é

$$
\mathfrak{n}(P)=\alpha t+\beta
$$

onde $\alpha$ e $\beta$ são constantes que podem, sem perda de generalidade, assumir os valores 1 e 0 , respectivamente.

As propriedades do conjunto dos instantes ficam, assim, condicionadas às características de $\mathfrak{n}$. Uma propriedade geral pode ser inferida de $e 1$. Qual seja, se existe um sistema físico tal que estabelecemos um ordenamento de $t$ por

$$
\mathfrak{n}(P)<\mathfrak{n}\left(P^{\prime}\right)<\mathfrak{n}\left(P^{\prime \prime}\right) .
$$

Logo, existe um processos físico equivalente, tal que podemos definir

$$
\mathfrak{n}\left(P^{\prime \prime}\right)<\mathfrak{n}\left(P^{\prime}\right)<\mathfrak{n}(P) .
$$

Este resultado encerra a reversibilidade da noção de tempo na mecânica, e é consequência da estrutura de $\mathfrak{G}$, em $e 1$, que é a de um grupo.

Outras características de $\mathfrak{n}$ podem se obtidas a partir da realização dos elementos constitutivos $e 1-e 5$. Vejamos como este esquema se aplica à mecânica Newtoniana e a mecânica relativística de Einstein. 


\subsection{Mecânica clássica não-relativística}

Os elementos constitutivos, de $e 1$ a $e 5$, são realizados na mecânica de Newton para pontos inerciais de massa $m$ (partículas) da seguinte maneira.

e1 - $\mathcal{V}=\mathbb{R}^{3} \times \mathbb{R}^{1}$, no qual $\mathbb{R}^{3}$ é o espaço Euclideano tridimensional, e $\mathbb{R}^{1}$ designará o tempo enquanto uma coordenada. Os sitemas inerciais $S$ são escolhidos como Galileanos, tais que os processos físicos analisados em $S$ e outro $\bar{S}$ estão relacionados por $\mathfrak{G}$ definido pelas Eqs. (3) e (4). Aqui é importante enfatizar que o tempo transcorre da mesma forma nos dois referenciais.

e2 - As variáveis mecânicas são descritas por funções reais, como o vetor posição $q \equiv \mathbf{x}(t)$, vetor velocidade $\mathbf{v}=\frac{d}{d t} \mathbf{x}(t)$, e momentum linear $\mathbf{p}(t)=m \frac{d}{d t} \mathbf{x}(t)$, onde $m$ é a massa.

e3 - O sistema físico é um ponto material de massa $m$. O estado é descrito por $\mathbf{x}(t)$, indicando a localização no espaço e no tempo, e $\mathbf{p}(t)$, descrevendo uma característica do movimento. Em geral uma variável mecânica, $F$ é descrita como uma função de $\mathbf{x}(t)$ e $\mathbf{p}(t)$, isto é $F=F(\mathbf{x}, \mathbf{p} ; t)$.

e4 - A Lagrangiana fica dada pela Eq. (2), com $q \equiv \mathbf{x}$.

e5 - A lei causal conectando os estados é estabelecida respeitando-se o elemento constitutivo $e 1$. Isto é, as transformações de Galilei servem de guia para se encontrar as leis que geram a dinâmica, que por vez deve ser consistente com o princípio de extremo dado na Eq. (1). Desse modo, usando métodos conhecidos em teoria de representações, resulta

$$
m \frac{d^{2} \mathbf{x}(t)}{d t^{2}}=\mathbf{F}(\mathbf{x})
$$

onde $\mathbf{F}$ é a força do meio sobre a partícula. Esta é a conhecida segunda lei de Newton. Note que esta equação não pode ser usada como definção de força, que é introduzida a partir de um processo de medida e fisicamente descreve a interação do meio com o sistema em observação.

Estamos considerando o problema de uma partícula por simplicidade; e pelo mesmo motivo, argumentos intuitivos têm prevalecidos ao rigor matemático [59].

Neste contexto Newtoniano, $\mathfrak{n}(P)$ possui, além das propriedades de ordemamento típicas da reta dos números reais, estrutura geométrica definida na reta (unidimensional). De fato, com a noção de distância entre dois instantes, denominada de intervalo de tempo, definida como a diferença entre dois pontos da reta real e com unidade fixa num dado sistema de referência, temos um espaço vetorial métrico. Isto significa que o conjunto dos instantes, é um espaço Euclidiano unidimensional, $\mathbb{R}^{1}$. Outrossim, devido a Eq. (4), a diferença de tempo entre dois instante é o mesmo para $\forall S$. Desta maneira, para dois sistemas $S$ e $\bar{S}$ temos

$$
\mathfrak{n}(P)-\mathfrak{n}\left(P^{\prime}\right)=\mathfrak{n}(\bar{P})-\mathfrak{n}\left(\bar{P}^{\prime}\right) .
$$

Neste contexto geométrico, então, as Eqs. (3) e (4) estão escritas na variedade $\mathbb{R}^{3} \times \mathbb{R}^{1}$, que é um espaço vetorial não métrico, a despeito dos subespaços $\mathbb{R}^{3}$ e $\mathbb{R}^{1}$ o serem.

\subsection{Mecânica clássica relativistica}

Na relatividade restrita os elementos constitutivos são dados por

e1 - $\mathcal{V}=\left\{x^{\mu}, g\right\}$ é o espaço de Minkowski $\mathbb{M}(3,1)$, onde a seguinte notação será empregada: $\left(x^{\mu}\right)$ é um 4-vetor $x=\left(x^{0}, x^{1}, x^{2}, x^{3}\right)=\left(x^{0}, \mathbf{x}\right)$, com $x^{0}=$ ct, $x^{1}=x, x^{2}=y, x^{3}=z$, sendo $c$ a velocidade da luz no vácuo; $g$, o tensor métrico, é tal que um elemento infinitesimal de distância em $\mathcal{V}$ é dado por

$$
(d s)^{2}=\left(d x^{0}\right)^{2}-(d \mathbf{x})^{2} .
$$

Neste caso $\mathcal{V}$ é do tipo dado no item anterior $\mathcal{V}=\mathbb{R}^{3} \times \mathbb{R}^{1}$, só que embebido de uma nova estrutura geométrica, caracterizada pela Eq. (17). Os referencias $S$ são ainda os referenciais inerciais como no item anterior. Entretanto, os processos físicos analisados em dois referenciais, um $S$ e outro $\bar{S}$, estão relacionados como já vimos antes pelo grupo de Lorentz 17]. As transformações de Lorentz $\Lambda^{\mu}{ }_{\nu}$ deixam $(d s)^{2}$, na Eq. (17), invariante. (De modo similar, uma formulação da mecânica não relativística baseada em espaços métricos pode ser introduzida a partir do grupo de Galilei, ver a Ref. 32 .)

Um caso particular de transfomações de Lorentz é dado por

$$
\begin{aligned}
\bar{t} & =\gamma\left(t-z v / c^{2}\right), \\
\bar{x} & =\gamma(x-v t), \\
\bar{y} & =y \mathrm{e} \bar{z}=z,
\end{aligned}
$$

$\operatorname{com} \gamma=1 / \sqrt{1-v^{2} / c^{2}}$. Estas equações descrevem o sistema $\bar{S}$ se deslocando com velociade $\mathbf{v}(|\mathbf{v}|=v)$ na direção do eixo $x$ do sistema $S$. Um aspecto relevante nessas transformações, com relaçãos às de Galilei, é que os processos físicos ficam restritos a um limite superior de velocidade, a velocidade da luz, $c[17,52$.

e2 - O conjunto de variáveis mecânicas são descritas, como no caso Newtoniano, por funções reais, definidas sobre $\mathcal{V}=\mathbb{R}^{3} \times \mathbb{R}^{1}$, mas agora, cabe frisar, como sendo um espaço de Minkowski. Assim temos, dentre outras, o 4-vetor posição $x^{\nu}(\tau)$; o 4-momentum dado por

$$
p^{\nu}(\tau) \equiv m \frac{d x^{\nu}(\tau)}{d \tau},
$$


onde $\tau$ é o tempo próprio, um invariante temporal definido por $\tau=d s / c$. Quando num referencial fixo, $\tau$ coincide com o tempo cronometrado por um relógio. As componentes de $p^{\nu}$ são as seguintes: $p=$ $\left(p^{0}, p^{1}, p^{2}, p^{3}\right)=(E / c, \mathbf{p})$, com $E$ sendo a energia.

e3 - O sistema físico será aqui descrito como um ponto material e o estado é dado, de modo similar ao caso não relativístico, por $x^{\nu}(\tau)$, indicando a localização no espaço-tempo, e $p^{\nu}(\tau)$ indicando um elemento de movimento.

e4 - A Lagrangiana é dada como antes (ver [17]).

e5 - A lei causal conectando os estados é estabelecida respeitando-se o elementos constitutivo $e 1$; isto é, as transformações de Lorentz servem de guia para o estabelecimento das leis que geram a dinâmica. O resultado é a generalização da segunda Lei de Newton

$$
m \frac{\partial x^{\nu}(\tau)}{\partial \tau}=f^{\nu}
$$

onde $f^{\nu}$ é a força relativística.

Agora estamos em posição de analisar as propriedades da coordenada tempo nas teorias Newtoniana e Einsteiniana.

Propriedade (i) - O tempo é uma coordenada definida sobre a reta Real nas duas teorias.

Propriedade (ii) - Processos descritos pelas Eqs. (13) e (14) são ainda válidos. Ou seja, como uma propriedade do grupos de Lorentz, a relatividade restrita é também reversível temporalmente.

Propriedade (iii) - Para sistema de referência fixo, o tempo próprio é definido como na Eq. 12 com unidade fixa. Então, este conjunto de instantes define, como no caso não-relativístico, um espaço Euclidiano unidimensional $\left(\mathbb{R}^{1}\right)$.

Propriedade (iv) - Devido às transformações de Lorentz, os intervalos de tempo não são os mesmos de um referencial para outro. Assim a Eq. (16) deixa de ser válida, e passa a ser regida por uma regra como aquela descrevendo a transformação dada pela Eq. (18)). Isto significa que os relógios passam de modo distinto de um sistema para o outro, contudo sem perder as regras de ordenamento. Usando a notação que enfatiza os processos podemos escrever

$$
\mathfrak{n}(\bar{P})-\mathfrak{n}\left(\bar{P}^{\prime}\right)=\gamma\left[\mathfrak{n}(P)-\mathfrak{n}\left(P^{\prime}\right)\right]
$$

Note que se $v<<c$, esta equação se reduz ao caso nãorelativístico da Eq. 16.

É importante ressaltar que em situações de altas velocidades, o limite superior de velocidade, a velocidade da luz, $c$, é estabelecido experimentalmente, de tal modo que um corpo massivo movendo-se com uma velocidade $v_{1}<c$ não pode ser acelerado até atingir uma velocidade $v_{2} \geq c$. A implicação básica disso é que, por motivos experimentais, não podemos lançar mão um sistema de referência inercial que se mova com velocidade $c$. Contudo não está proibido a existência de objtos se movendo com velocidades superior a $c$; os chamados táquions. Com as noções de espaço e tempo assim definidas, considera-se a existência dos sistemas inerciais, levando às transformações de Lorentz.

\section{Teoria cinética clássica relativística}

Nesta seção, a teoria cinética relativísitica é elaborada como uma teoria mecânica enfatizando sua estrutura, que é a de uma teoria de campos. Embora seguimos, preocupados também por razões pedagógicas, uma apresentação não usual, outros aspectos complementares podem ser encontrados em outros textos muito bem elaborados 60 63.

Os sistemas de refêrência inerciais são especificados pelo grupo de Lorentz e o sistema físico será um gás carregado (um plasma) relativístico. Esse sistema é especificado por um campo definido no espaço de fase, provendo uma densidade de probabilidade em cada ponto.

Há várias formas de se introduzir o espaço de fase, que é a variedade natural (experimentalmente consistente) para tratar o sistema físico em questão, um plasma clássico relativísitico 63. Seguiremos uma apresentação matemática geral 64 .

Considere $\mathbb{M}$ o espaço Minkowski onde cada ponto é especificado pela coordenada $q^{\mu}$, com $\mu=0,1,2,3$. O correspondente espaço cotangente, $T^{*} \mathbb{M}$, é especificado por um conjunto duplicado de coordenadas $\left(q^{\mu}, p^{\mu}\right)$, que pode ser equipado com uma estrutura simplética definida por uma 2-forma (uma estrutura métrica tensorial de grau 2) dada por $\omega=d q^{\mu} \wedge d p_{\mu}$

$$
\Lambda=\frac{\overleftarrow{\partial}}{\partial q^{\mu}} \frac{\vec{\partial}}{\partial p_{\mu}}-\frac{\overleftarrow{\partial}}{\partial p^{\mu}} \frac{\vec{\partial}}{\partial q_{\mu}}
$$

Considerando as funções $C^{\infty} f(q, p)$ and $g(q, p)$, tem-se

$$
\omega(f \Lambda, g \Lambda)=f \Lambda g=\{f, g\}
$$

em que $\{f, g\}=\frac{\partial f}{\partial q^{\mu}} \frac{\partial g}{\partial p_{\mu}}-\frac{\partial f}{\partial p^{\mu}} \frac{\partial g}{\partial q_{\mu}}$ é o parênteses de Poisson. O espaço $T^{*} \mathbb{M}$ equipado com esta métrica simplética é chamado de espaço simplético ou espaço de fase, $\Gamma$.

Introduzindo a seguinte notação $\omega=\left(\omega^{1}, \omega^{2}, \ldots, \omega^{8}\right)$, $\operatorname{com} \omega^{1}=q^{0}, \omega^{2}=q^{1}, \omega^{3}=q^{2}, \omega^{4}=q^{3}, \omega^{5}=p^{0}, \omega^{6}=$ $p^{1}, \omega^{7}=p^{2}, \omega^{8}=p^{3}$ tal que $q=\left(q^{0}, \mathbf{q}\right)$ e $p=\left(p^{0}, \mathbf{p}\right)$ 4 -vetores em $\mathbb{M}$, a métrica simplética em $\Gamma,\left(\eta_{a b}\right), a, b=$ $1, \ldots, 8$, reduz-se a

$$
\eta=\left(\begin{array}{cc}
0 & I \\
-I & 0
\end{array}\right)
$$

Desse modo, dado duas funções $f(\omega)$ and $g(\omega)$ of class $C^{\infty}$, o produto escalar é escrito como

$$
\omega(f \Lambda, g \Lambda)=\eta^{a b} \frac{\partial f}{\partial \omega^{a}} \frac{\partial g}{\partial \omega^{b}}=\eta^{a b} \partial_{a} f \partial_{b} g .
$$


Essa estrutura é bem conhecida em mecànica clássica na realização de Hamilton-Poisson da relatividade restrita. Entretanto, $\Gamma$ como introduzido aqui é matematicamente geral, e pode ser utilizado em outros contextos. No caso da teoria cinética, o sistema é caracterizado por um campo $f(q, p) \in \Gamma$ que é uma densidade de probabilidade. Para descrever efeitos de correlações entre pontos distintos no espaço se introduz as densidade de probabilidades a n-pontos (ou n-corpos), $f_{N}=f_{N}\left(q_{1}, \ldots, q_{N}, p_{1}, \ldots, p_{N}\right)$. Aqui consideraremos apenas as distribuições a 1-ponto.

O estado desse sistema é caracterizado então por $f(q, p)$ e suas derivadas com relação aos 4 -vetores $q$ e $p$. A função $f(q, p)$ é introduzida de tal modo a respeitar a conservação de probabilidade no espaço de fase relativístico. Assim, $f(q, p)$ deve satisfazer, além da normalização, o teorema de Gauss da 4-divergência, isto é,

$$
\int d^{4} p d^{4} q \frac{\partial}{\partial q^{\mu}} f(q, p) p^{\mu}=0 .
$$

Similar resultado deve valer para a divergência em $p$. Esta função fica associcada à função de distribuição clássica não relativística, $f_{c}(\mathbf{q}, \mathbf{p}, t)$, através da integração

$$
f_{c}(\mathbf{q}, \mathbf{p}, t)=\int d p^{0} f(q, p)
$$

Neste caso, $f_{c}(\mathbf{q}, \mathbf{p}, t)$, que é também Lorentz-invariante, se reduz trivialmente ao caso não-relativístico.

Uma simples situação de evolução de $f(q, p)$ é obtida a partir de uma densidade de Lagrangiana dada por

$$
\mathcal{L}=\rho(q, p)\left[p^{\mu} \frac{\partial}{\partial q^{\mu}}+F^{\mu} \frac{\partial}{\partial p^{\mu}}\right] f(q, p)+\frac{1}{2} \lambda \rho^{2},
$$

em que $F^{\mu}=p_{\nu} F^{\nu \mu}$ é a força de Lorentz, com $F^{\nu \mu}$ sendo o (antissimétrico) tensor intensidade de campo eletromagnético, $F^{\mu \nu}=\partial^{\mu} A^{\nu}(q)-\partial^{\nu} A^{\mu}(q)$, tal que $\partial_{\mu}=\partial / \partial q^{\mu}$ e $A^{\mu}(q)$ é o 4 -vetor potencial. Note que a Lagrangiana dada na Eq. (26) é basicamente o acoplamento do campo $f(q, p)$ com uma corrente externa definida através da parte espacial e a parte do momento, isto é, $j_{q}^{\mu}=\rho p^{\mu}$ and $j_{p}^{\mu}=\rho F^{\mu}$. O acoplamento ocorre então através de dois termos, $j_{q}^{\mu} \frac{\partial}{\partial q^{\mu}} f(q, p)$ e $j_{p}^{\mu} \frac{\partial}{\partial p^{\mu}} f(q, p)$. Isto conduz à equação de Euler-Lagrange

$$
p^{\mu} \frac{\partial}{\partial q^{\mu}} f(q, p)+F^{\mu} \frac{\partial}{\partial p^{\mu}} f(q, p)=\lambda \rho,
$$

que é uma equação do tipo Vlasov relativística com uma fonte externa, $\rho$, satisfazendo

$$
p^{\mu} \frac{\partial}{\partial q^{\mu}} \rho(q, p)+F^{\mu} \frac{\partial}{\partial p^{\mu}} \rho(q, p)=0 .
$$

Dado a arbitrariedade da fonte, é possível considerar neste ponto $\rho$ constante (independente de $q$ e $p$ ) e $\lambda=0$. Assim chega-se à equação de Vlasov usual para um plasma na presença de um campo eletromagnético externo 60, 63, tal que $p^{\mu} \frac{\partial}{\partial q^{\mu}} f(q, p)$ expressa a evolução livre da densidade de probabilidade, que é conservada no espaço de fase. Em termos outros, considerando explicitamente as componentes do tensor de campo $F^{\mu \nu} \mathrm{e}$ a Eq. 25), temos

$$
\frac{\partial}{\partial t} f_{c}(q, p)+\mathbf{v} \cdot \frac{\partial}{\partial \mathbf{q}} f_{c}(q, p)+\mathbf{F} \cdot \frac{\partial}{\partial \mathbf{p}} f_{c}(q, p)=0,
$$

com

$$
\mathbf{F}=e \mathbf{E}+\frac{e}{c} \mathbf{V} \times \mathbf{B}
$$

onde $\mathbf{E}$ and $\mathbf{B}$ são os campos elétricos e magnéticos, respectivamente, $e$ é a carga elétrica e $c$, a velocidade da luz.

É possível ainda introduzir um termo de colisão não linear em $f(q, p)$. Neste caso, a Lagrangiana pode ser acrescida de um termo como $\mathcal{L}_{\text {Colis. }}=\rho C[f]$, tal que, a equação de movimento ( $\operatorname{com} \lambda=0$ e $\rho$ constante) fica dada por

$$
p^{\mu} \frac{\partial}{\partial q^{\mu}} f(q, p)+F^{\mu} \frac{\partial}{\partial p^{\mu}} f(q, p)=\mathcal{C}[f] .
$$

A escolha adequada do termo de colisão conduz, por exemplo, à equação de Boltzmann. Todavia, esse tipo de escolha, que resulta ser compatível com a segunda lei da termodinâmica, leva a uma quebra de simetria de Lorentz, e assim a uma redefinição do primeiro elemento constitutivo. De fato, para satisfazer a lei de crescimento da entropia, a irreversibilidade temporal é imposta, de tal modo que transformações inversas no tempo, uma característica da definição de grupo, não são mais possíveis. Neste caso se tem um semi-grupo. Os outros elementos constitutivos seguem como antes e são facilmente enumeráveis, em particular o mapeamento nos reais, para efeito de medida, estabelecido pela média dos observáveis.

Para terminar é importante frisar alguns aspectos. Em teoria cinética, é comum se referir a $f(q, p)$ como o estado do sistema, por exemplo, o estado de um plasma 62 . Mas esta nomenclatura não é consistente do ponto de vista de uma teoria do movimento, na medida em que se trata de um campo. Pelo mesmo motivo que devemos nos referir a $A^{\mu}(q)$ e suas derivadas como o estado do campo eletromagnético, a partir do qual a densidade de Lagrangiana é escrita, então $f(q, p)$ e também suas derivadas, $\partial_{q} f(q, p)$ e $\partial_{p} f(q, p)$, são quem estabelece o estado do sistema descrito pela função de distribuição. Outro aspecto relacionado a terminologia é o frequente uso do conceito de ponto material para os propósitos de raciocínio ao se deduzir, por exemplo, os termos de colisão do tipo Boltzmann; e isso é muito útil. Entretanto, há de se ter em mente que, basicamente, a teoria cinética é uma mecânica de campo clássico (uma generalização do campo de velocidade), com as devidas funções de correlações entre um ponto e outro do espaço de fase.

\section{Conclusões finais}

Considerando elementos constitutivos, a noção de mecânica, ou seja, de uma teoria do movimento, é analisada conjuntamente com uma definição de mensuração. O 
ponto de partida é a investigação das propriedades do conceito de tempo e espaço, seguido da conceitualização de estado mecânico, ponto material e campos. Neste contexto, as relações entre as mecânicas relativística e não-relativística de um ponto material são tratadas. A construção é ainda aplicada na formulação da teoria cinética relativística, enquanto uma teoria de campo. No decorrer do texto, vários aspectos pedagógicos foram sendo discutidos, em particular a definição de tempo e espaço, e as suas características geométricas.

Um elemento central desta abordagem é a perspectiva de que o tempo é definido e utilizado em física a partir de relações entre objetos físicos que estabelecem o movimento. Ou seja, na física, o tempo existe enquanto processos e relações, considerados como elementos ontológicos, a partir dos quais se introduz a medida. Neste cenário, o conceito de tempo absoluto de Newton foi analisado.

O tempo absoluto é usualmente considerado um atributo característico da física não-relativística. Corrobora a esta opinião o fato de que o tempo transcorre da mesma forma nos diversos sistemas de referência, como consequência da Eq. (16). Todavia, tanto na mecânica de Newton como na de Einstein, o tempo é definido a partir de relações (ver a definição de $\mathfrak{n}(P)$ na seção 6 ), e é assim, para todos os fins práticos, de mesma natureza; diferindo nas qualidades associadas ao grupo de invariância da teoria (compare as Eqs. (16) e (22)). Ou seja, o tempo na relatividade continua a ser ainda o tempo das relações estabelecidas a partir do padrão de medida, o relógio. Mas para tratar a sincronicidade, atributos outros, como dilatação e contração, precisaram ser incorporados à definição original do tempo, quando nas escalas das velocidadess altas.

Essas conclusões, obtidas da análise dos elementos constitutivos de uma teoria sobre o movimento, são estabelecidas pelo eixo escolhido pela física para proceder no seu desenvolvimento: o experimento. Isso independe, em certa medida, do ponto de vista de seus agentes, quer sejam realistas, ou românticos ou ainda metafísicos transcedentes; mas também não minimiza ou nega a importância dos elementos culturais, como o pensamento realista e a lógica na criação de teoria físicas. O que, em última instância, se impôe para a práxis da física é o experimento, que tem como ponto de partida as relações entre os objetos físicos, em qualquer escala de observação. A opção pelo eixo experimental é nítida no processo que levou à elaboração da física do Sec. XX.

Um exemplo é a relatividade restrita. Nesse caso, observa-se que o movimento possui características próprias associadas com as escalas de velocidade. Isso é o que se constata, experimentalmente, no regime de altas velocidades, com o limite da velocidade da luz, e que levou à mecânica relativística. O processo histórico não foi linear [52], mas ainda assim é importante observar que a construção da relatividade teve como premissas o experimento, a definir a escolha de caminhos filosóficosinterpretativos. Por exemplo, a conceito de éter, tão estimado e caro à física do Sec. XIX, fora completamente abandonado 52 .

Uma outra amostra é a física quântica. Nesse caso microscópico, i.é, no âmbito atômico e subatômico, o movimento se processa de tal modo que ao se medir as suas características, aspectos próprios dessas escalas de comprimento (ou seja, aspectos que não estão presentes na mecânica de Newton) surgem. Isso levou aos elementos fundamentais daquilo que caracteriza o movimento na escala quântica: as relações experimentais de incerteza de Heisenberg. Essa constação, mais uma vez, não diminui nem releva o imenso esforço intelectual de físicos e filósofos para estabelecer e criar quadros conceituais que conduziram à mecânica quântica. Discussão que ainda continua, mas encontra as devidas escolhas teóricas no experimento. Vale mencionar os modelos de partículas ocultas, que, embora intelectualmente interessantes, não resistiram as demandas experimentais [4].

As concepções de mundo, em perspectiva cultural, têm sido um instrumento formidável na construção das teorias do movimento. Contudo, dos dois exemplos citados acima, constata-se que não é suficiente "um bom conceito", nem qualquer tipo de ontologia. Por vezes, certas concepções culturais guiam a construção de teorias. Esse é o caso, mas ainda em elaboração, e pouco questionado, do modelo padrão cosmológico, com o seu conceito de início, aceito em quase uníssono, mas ainda de frágil teste experimental. Outros elementos aparecem na mesma esteira de guias, como as célebres afirmativas de que o "mundo é matemático", ou ainda "a física é geométrica, ou topológica", entre outras 65]. Essas afirmativas remetem a elementos ontológicos do mundo. Mas em sua práxis, a física em algum momento há de perguntar-se: "Qual matemática?, Qual geometria?, Qual topologia?". A pergunta, que permanece válida no âmbito do experimento, desmonta o caráter ontológico da assertiva. O que se pode concluir é que a práxis da física guarda compromisso apenas com as ontologias que fundam os procedimentos de medida. Sob essa perspectiva, as chamadas teorias de tudo (do Inglês TOE: theory of everything) podem ser vistas como falácias naturalistas, que, as vezes, no discurso midiático da divulgação científica equivocada, se transmudam nas denominadas falácias do espantalho.

Ainda é necessário apontar, embora preliminarmente, que as expectativas pós-modernas da nova metodologia científica do "paradigma emergente" 66 não ocorreram por completo. Essas noções foram formuladas, pelo menos no que se refere a áreas da ciências naturais, considerando novos conceitos introduzidos pela relatividade, mecânica quântica e vários aspectos da teoria cinética e da física estatística do não equilíbrio nas décadas de 1970 e 1980, que deram origem à denominada física dos sistemas complexos. Entretanto, como vimos aqui neste trabalho, a essência metodológica da física contemporânea, mesmo com uma imensa gama de novos fenômenos e conceitos, continua a ser fundamentalmente aquela Newtoniana; não ocorreram mudanças metodológicas tão radicais. Mas o que parece ser interessante é que noções como a de tempo e espaço, na apropriação da física desde Newton, já se 
revelavam mais como conceitos de uma contrução social [51] que filosófica. Em um próximo trabalho, esses aspectos, assim como a estrutura dos elementos constitutivos da mecânica quântica e das teorias quânticas de campos, serão abordados com mais detalhe.

\section{Agradecimentos:}

Este trabalho teve apoio financeiro do $\mathrm{CNPq}$.

\section{Referências}

[1] M. Jammer, Concepts of Space (Dover, New York, 1954).

[2] M. Jammer, The Philosophy of Quantum Mechanics: The interpretations of QM in Historical Perspective (John Wiley, New York, 1974).

[3] O. Freire Jr., O. Pessoa Jr. e J.L. Bromberg (Orgs.), Teoria quântica: Estudos Históricos e Implicações Culturais (EDUEPB e Livraria da Física Editora, Campina Grande e São Paulo, 2011).

[4] O. Freire Jr., David Bohm e a Controvérsia dos Quanta (CLE/Unicamp, Campinas, 1999).

[5] O. Freire Jr. in: A Cultura da Fúsica: Contribuiçôes em Homenagem a Amélia Hamburger, editado por A.A.P. Videira e S.R.A. Salinas (LF Editorial, São Paulo, 2001), p. 51.

[6] O. Freire Jr., in: Origens e Evolução das idéias da Física editado por J.F. Rocha (EDUFBA, Salvador, 2002), p. 285.

[7] A. Ribeiro-Filho, in: Origens e Evolução das Idéias da Física editado por J.F. Rocha (EDUFBA, Salvador, 2002), p. 285

[8] J.C.A. Barata, in: 100 Anos da Física Quântica editado por M.S. Hussein e S.R.A. Salinas (LF Editorial, 2002), p. 33.

[9] O. Freire Jr., The Quantum Dissidents: Rebuilding the Foundations of Quantum Mechanics (1950-1990) (Springer, Berlin, 2015).

[10] A.F.R.T. Piza, in: 100 Anos da Física Quântica editado por M.S. Hussein e S.R.A. Salinas (LF Editorial, São Paulo, 2002), p. 13.

[11] P.A. Nusseinzveig, in: 100 Anos da Física Quântica editado por M.S. Hussein, S.R.A. Salinas (LF Editorial, São Paulo, 2002), p. 101.

[12] M. Bondani, I.P. Degiovanni, M. Genovese, M.G.A. Paris, I.R. Berchera e V. Schettini, Found. Phys. 41, 305 (2011).

[13] R. Omnès, Found. Phys. 43, 1339 (2013).

[14] M. Fox, Quantum Optics, an Introduction (Oxford University Press, Oxford, 2006).

[15] A. Di Domenico, A. Gabriel, B.C. Hiesmayr, F. Hipp, M. Huber, G. Krizek, K. Mühlbacher, S. Radic, C. Spengler, L. Theussl, Found. Phys. 42, 778 (2012).

[16] J.A. Helayel-Neto, L.P.R. Ospedal, in: III Escola de Física Roberto Salmeron editado por A.E. Santana, R.G.G. Amorin, S.C. Ulhoa, V.C. Andrade, A. Ibaldo, A.L.A. Penna, E.R. Nunes e J.D.M. Vianna (LF Editorial, São Paulo, 2017), v. 1, p. 19.

[17] S. Weinberg, Gravitation and Cosmology (John Wiley, New York, 1972).

[18] I. Umezawa, Math. Japonica 41, 109 (1995).

[19] M.A. Moreira, Rev. Bras. Ens. Fis. 31, 1306 (2009).
[20] F.K. Nóbrega e L.F. Mackedanz, Rev. Bras. Ens. Fis. 35, 1301 (2013).

[21] M.E. Perskin e D.V. Schröder, An Introduction to Quantum Field Theory (Addison-Wesley, New York, 1995).

[22] L.D. Landau e E.M. Lifshitz, Quantum Mechanics (Pergamon, London, 1959).

[23] E. Comay, Prog. Phys. 4, 91 (2009).

[24] A.V. Selikov e M. Gyulassy, Phys. Rev C 49, 1726 (1994).

[25] F.C. Khanna, A.P.C. Malbouisson, J.M.C. Malbouisson e A.E. Santana, Phys. Rep. 539, 135 (2014).

[26] E.P. Wigner, Ann. Math 40, 149 (1939).

[27] F.C. Khanna, A.P.C Malbouisson, J.M.C. Malbouisson e A.E. Santana, Thermal Quantum Field Theory: Algebraic Aspects and Applications (World Scientific, Singapore, 2009).

[28] R.D. Sorkin, ArXiv:gr-qc/9401003 (1994).

[29] N.C.A. da Costa e O. Bueno, in: A Cultura da Física: Contribuições em Homenagem a Amélia Hamburger editado por A.A.P. Videira e S.R.A. Salinas (LF Editorial, São Paulo, 2001), p. 51.

[30] H.Q. Plácido, R. Bunchaft, A.E. Santana e Hadronic J. 15, 225 (1992).

[31] A.E. Santana, in: Proceedings da Semana de Geologia da UEFS, Feira de Santana, 1996, editado por L.M. Barbosa (DEXA, área de geologia, UEFS, Feira de Santana, 1996).

[32] A.E. Santana, Rev. Bras. Ens. Fis. 19, 113 (1997).

[33] A.E. Santana, F.C. Khanna e Y. Takahashi, Prog. Theor. Phys. 99, 327 (1998).

[34] L.A.C. Malbouisson e A.E. Santana, Ciência e Sociedade 2, 8 (2014).

[35] A.E. Santana e S. Simon, J. Mod. Phys. 6, 58 (2015).

[36] K. Berka, Measurement: Its Concepts, Theories and Problems (D. Reidel, Dordrecht, 1983).

[37] D.S. Lands, Revolution in Time (Harvad University Press, London, 1983).

[38] I. Newton, The Principia (Prometheus Books, New York, 1995).

[39] J. Needham, W. Ling e D.J.S. Price, Heavenly Clockwork: the Great Astronomical Clocks of Medieval China (Cambridge University Press, Cambridge, 1960).

[40] P. Davies, About Time: Einstein's Unfinished Revolution (Simon\&Schuster, London, 1995).

[41] G.J. Whitrow, O Tempo na História (Jorge Zahar, Rio de Janeiro, 1988).

[42] D. Sobel, Longitude (Ediouro, Rio de Janeiro, 1996).

[43] M.R. Matthews, Time for Science Education: How Teaching the History and Philosophy of Pendulum Motion Can Contribute to Science Literacy (Springer, New York, 2000).

[44] J.L. Synge, Talking About Relativity (North-Holland, Amsterdan, 1970).

[45] K.G. Denbigh, Three Concepts of Time (Springer-Verlag, Berlim, 1981).

[46] W.H. Newton-Smith, The structure of Time (Routledge and K. Paulo, London, 1980).

[47] I. Prigogine e I. Stengers, La Nouvelle Alliance (Gallimard, Paris, 1979).

[48] I. Prigogine, O Fim das Certezas: Tempo, Caos e as Leis da Natureza (Editora Unesp, São Paulo, 1996).

[49] A. Pereira Jr., Irreversibilidade Física e Ordem Temporal na Tradição Boltzmanniana (Editora Edusp, São Paulo, 1997). 
[50] St. AgostinhoConfissões, Livro XI, O Homem e o Tempo (N. Cultural, São Paulo, 1996).

[51] N. Ellias, Sobre o Tempo (Jorge Zahar, Rio de janeiro, 1984).

[52] A.M.M. Polito, A Construção da Estrutura Conceitual da Física Clássica (LF Editorial, São Paulo, 2016).

[53] J.F.M. Rocha, Rev. Bras. Ens. Fis. 31, 1604 (2009).

[54] L.D. Landau e E.M. Lifshitz, Mécanique (Mir, Moscou, 1966).

[55] E.C.G. Sudarshan e N. Mukunda, Classical Dynamics: A modern Perspective (J. Wiley, New York, 1974).

[56] H.B. Callen, Thermodynamics and an Introduction to Thermostatistics (J. Wiley and Sons, New York, 1985).

[57] M.J. Oliveira, Termodinâmica (LF Editorial, São Paulo, 2005).

[58] J.D. Jackson, Classical Electrodynamics (J. Wiley, New York, 1962).

[59] S.F. Cortizo, Rep. Math. Phys. 29 (1991) 45.

[60] M. Le Bellac, F. Mortessagne e G.G. Batrouni, Equilibrium and Non-equilibrium Thermodynamics (Cambridge University Press, Cambridge, 2004).

[61] T. Tomé e M.J. Oliveira, Stochastic Dynamics and Irreversibility (Springer-Verlag, Berlin, 2015).

[62] R. Balescu, Statistical Dynamics: Matter oyut of Equilibrium (Imperial College Press, London, 1997).

[63] R.L. Liboff, Kinetic Theory: Classical, Quantum and Relativistic Description (J. Wiley, New York, 1998).

[64] C. Costa, M.R. Tenser, R.G.G. Amorim, M.C.B. Fernandes, A.E. Santana e J.D.M. Vianna, Advances in Applied Clifford Algebras 28, 27 (2018).

[65] H. Weyl, Symmetry (Princeton Press, Princeton, 1980).

[66] B.S. Santos, Um Discurso Sobre as Ciências (Cortez Editora, São Paulo, 2018). 